\title{
Análisis de la situación de maltrato sufrido fuera de la institución por personas mayores en el contexto de la atención residencial
}

\author{
Carmelo Gómez Martínez ${ }^{1,2}$, Elena Carrasco Martínez ${ }^{1}$, Inmaculada Martínez \\ Escámez ${ }^{1}$ y Pedro Andreo Muñoz ${ }^{1}$ \\ ${ }^{1}$ Asociación Edad Dorada Mensajeros de la Paz Murcia (España); \\ ${ }^{2}$ Universidad Católica San Antonio de Murcia (España)
}

\begin{abstract}
Los ancianos son más vulnerables y determinados condicionantes socioculturales hacen que el maltrato sea poco evidenciado y por ello, poco abordado. Dentro del segmento poblacional de personas mayores, se dan las circunstancias concretas para que se dé el maltrato. Nuestra aportación a la hora de abordar esta cuestión ha sido conocer el número y tipo de casos de maltrato que los ancianos que viven en residencias y que sufren por agentes ajenos a la organización de la misma, tales como la familia, otros residentes y amigos. Para este estudio se ha elaborado un breve cuestionario en una hoja de Excel para la recogida de datos, que ha sido pasado a las trabajadoras sociales de 4 residencias de personas mayores, en el ámbito geográfico de la Región de Murcia. Los datos recogidos corresponden al periodo de tiempo comprendido entre enero y diciembre de 2013. Los resultados obtenidos nos llevan a pensar que los principales factores de riesgos asociados a este tipo de maltrato es ser mujer, viuda y con una edad de aproximadamente 80 años. El maltrato psicológico ejercicio sobre todo por familiares es el más frecuente.
\end{abstract}

Palabras clave: Maltrato, residencia, no institucional, ancianos.

Analysis of the situation of abuse suffered outside the institution older people in the context of residential care. Within the elderly population, the specific circumstances that some of the elderly find themselves due to certain socio-cultural factors make them more likely to be abused while residing in assisted living residences, due to the facts that the abuse is coming from the organisation it is unlikely to be reported, or brought to the attention of others, making it difficult to detect and, as a result, an issue not often discussed. We intend to investigate these possibilities; however, due to lack of information on this subject, we aim to address this issue by investigating the number of elderly people living in nursing homes who have suffered abuse due to agents outside of the organisation, such as the family, other residents and Friends. A brief questionnaire was created on an Excel Spreadsheet for the collection of data for this study, these questionnaires where then passed on the social workers of 4 assisted living residences, in the geographical scope of the Murcia region. The data collected correspond to the time period between January and December 2013. The results lead us to believe that the principal risk factors associated with this type of abuse is being a woman, a widow and with and age of about 80 years. Psychological abuse from family members was the most frequently reported type of abuse in the study.

Key words: Abuse, residence, non-institutional, elderly.

Correspondencia: Carmelo Gómez Martínez. Asociación Edad Dorada Mensajeros de la Paz Murcia. C/ Orden de Santiago, 5. Abarán, Murcia (España).

E-mail: coordinacionregional@mensajerosdelapazmurcia.es 
El objetivo principal de este trabajo es conocer la existencia y tipo de maltrato recibido por las personas mayores que viven en residencias, por parte de agentes no institucionales.

\section{Desarrollo del tema}

La atención a personas mayores es un tema no exento de polémica teniendo en cuenta las variables socioculturales de la rodean. Nuestra sociedad ha ido cambiado poco a poco, y como consecuencia del progreso tecnológico y social, los modelos de atención y cuidado de las personas mayores. Así, de un modelo asistencial que se basaba históricamente en la familia tradicional, donde podían llegar a convivir tres generaciones al mismo tiempo en una casa garantizando de este modo el cuidado de los más vulnerables (ancianos y niños), se ha pasado a un modelo asistencial delegado en terceros, ya sean físicos o institucionales, producto de la familia mononuclear, con dos generaciones en el hogar en el que los progenitores trabajan y los hijos no suelen pasar de dos de promedio; esta última situación pone en serio riesgo la continuidad del cuidado de los mayores y los niños dentro del núcleo familiar.

Así, se hace necesario delegar los cuidados de los mayores en instituciones, de mayor o menor rango (residencias y centros de día respectivamente), convirtiendo a estas en sustitutas de la familia. Esto no debe ser entendido como una ruptura total ya que si bien la institución residencial como tal se hace presente a través de normas y deberes, estas están debidas a proporcionar la mayor independencia y autonomía a las personas que en ella viven. La familia solo delega una parte del cuidado, el profesional, pero no delega la calidad de la relación interpersonal que es establecida entre persona mayor y resto de familia, antes del ingreso de este en la residencia.

Esto debe ser no solo entendido por los miembros de la institución residencial sino que debe ser promovido y mejorado, cuando sea posible. La autonomía de la persona mayor debe ser la base del paradigma asistencial social (Rodríguez, 2007).

Este enfoque no está exento de sus riesgos desde el punto de vista institucional ya que si bien es cierto que normativamente (Comunidad Autónoma de la Región de Murcia, 2005) es responsabilidad de la dirección del centro el promover la participación y la autonomía de los mayores que viven en las residencias y centros de día, cuando es vulnerada por agentes externos al personal de la residencia, mientras en anciano tiene su estatuto de "residente" no deja claro cómo proceder.

La experiencia nos dice que en algunas ocasiones el día a día de la organización de un servicio de las características de una residencia y/o un centro de día es tan complejo que nos podemos ver inmersos de manera "focalizada" a solucionar exclusivamente los problemas derivados de la relación entre la entidad que presta el servicio y el anciano y la familia que los recibe. Pero, ¿qué pasa cuando el mayor, en su estatuto de residente, es objeto de tratos no dignos, e incluso delictivos, por personas 
ajenas a la propia organización de la residencia pero mientras vive institucionalmente en esta?. La respuesta a esta pregunta no está en bibliografía alguna ya que la pregunta no suele hacerse de manera sistemática. Es muy relevante el hacerse esta cuestión ya que existen situaciones que si bien escapan a la reglamentación ordinaria no por ello pueden ser obviadas. Hablamos en el caso que nos ocupa de una situación a la que se ven sometidos los ancianos residentes de una residencia por la especial vulnerabilidad que muestran. Esta citada vulnerabilidad constituye una consecuencia de factores biológicos, psicológicos y sociales relacionados con el envejecimiento. Todos estos factores pueden constituir un contexto en el cual el "yo" de la persona mayor se vea martirizada por las nuevas normas y condicionantes formales e informales de la propia institucionalización (Goffman, 1970).

Quizás un grupo de factores importante lo constituyan las cuestiones socioculturales, ya que estas condicionan en gran medida la percepción que los mayores tienen del mundo que les rodea. Por ejemplo, y en relación al objeto de la presente investigación, el maltrato físico, o violencia de género, es una situación "normalizada" por mujeres que vivieron en contextos sociales donde el papel de la mujer era de subalternidad respecto al del hombre (Espinella, 2013); así una bofetada, o un empujón podía verse como un gesto de "poner las cosas en su sitio" en momentos donde la mujer protagonizaba una salida de contexto, tal y como el hombre, y la sociedad, lo entendía. Evidentemente, ni todos los casos son tal y como hemos descrito, ni estos se dan en todas las mujeres y hombres de edad avanzada.

La inquietud del presente trabajo estriba en hacer visible lo intuido por los que de un modo u otro trabajan en el mundo asistencial residencial, respecto al ejercicio de hegemonía sobre una persona mayor, y que suponga un perjuicio para este último. Concretando, nos interesa conocer si realmente el anciano es sometido a algún tipo de vejación ya sea física, psicológica, sexual o cuantas formas podamos entender y que vienen claramente descritas al efecto (AAVV, 2007; Espinella, 2013). Siendo más precisos, nos interesa mucho conocer hasta qué punto estas supuestas vejaciones son ejercidas por miembros de la familia, en mayor o menor grado de parentesco, ya que la familia puede ser un foco de maltrato cuando la acción de cuidar se vuelve patológica para el que cuida (Delgado, 2012). También nos interesa conocer cuáles son ejercidas por otras personas que no son de la organización que forma la entidad que acoge institucionalmente al anciano.

Todas estas características nos han permitido conocer, admitimos que de manera descriptiva y exploratoria, la situación de maltrato del que una persona mayor es objeto siendo este más o menos consciente de ello. 


\section{MÉTODO}

\section{Sujetos}

Para poder recoger los datos se ha establecido un primer grupo de profesionales, que si bien no se ha constituido en un focus-group nos ha permitido intuir la mayor o menor evidencia del problema. Este grupo estaba formado tanto por los miembros de la dirección de cada residencia perteneciente al estudio, como las trabajadoras sociales de cada una de estas. En total han sido ocho las personas con las cuales se celebró una primera reunión. La población objeto de este trabajo fue la institucional residencial de la Región de Murcia, recogiendo los datos en aquellas que por facilidad de acceso al campo de estudio nos abrieran las puertas a esta misión. Al final la población objeto del estudio la compuso un total de 368 personas mayores que vivían en residencias, todas estas últimas gestionadas por la Asociación Edad Dorada Mensajeros de la Paz Murcia. El trabajo de investigación se diseñó y se planteó al escrutinio del Comité de Ética Asistencial de dicha Asociación no encontrando conflicto ético alguno.

\section{Instrumentos}

El formato que se utilizó para ordenar los datos y posteriormente procesarlos fue una tabla de Excel, del paquete de Microsoft.

\section{Procedimiento}

Como resultado de esta se preparó un breve cuestionario que pretendía recoger una serie de datos, para su posterior análisis cuantitativo. También se elaboró un listado preliminar que incluyó aquellas personas mayores que tanto las directoras como las trabajadoras sociales sospechaban con mayor o menor evidencia que recibían o habían recibido algún tipo de maltrato. Las categorías en las cuales agrupamos los ítems fueron las siguientes: datos de filiación (edad y sexo), tipo de maltrato al que había estado o estaba siendo objeto (físico, sexual, negligencia, abandono, psicológico, económico), momento en el cual se dio o se estaba dando (antes, después o durante el ingreso) y por último, por parte de quien provenía el maltrato (hijos, cónyuge, otra familia y otros no familiares). La forma en que fueron recogidos los datos fue preguntándole directamente a los mayores. Estas recogida fue realizada por las trabajadoras sociales ya que ellas tenían ganada la confianza de los mayores y el acceso a este tipo tan delicado de datos gozaba de bastante garantía. El periodo de tiempo destinado a ubicar la situación del maltrato fue desde Enero hasta Diciembre del año 2013. El proceso de análisis de los datos fue sencillo ya que partiendo de la premisa de que este ha sido un trabajo de investigación de carácter exploratorio solo buscábamos 
conocer las prevalencias. En función de los resultados se podría plantear en un futuro un trabajo más exhaustivo desde el punto de vista analítico.

\section{RESULTADOS}

Del total de personas mayores potenciales de entrevistar, preliminarmente se seleccionaron por las trabajadoras sociales 36 sujetos. Los resultados fueron los siguientes:

\section{Edad de la persona maltratada}

La edad promedio de la persona mayor maltratada es de 80 años, siendo el límite inferior de 61 años y el superior de 94 años.

\section{Sexo de la persona mayor maltratada}

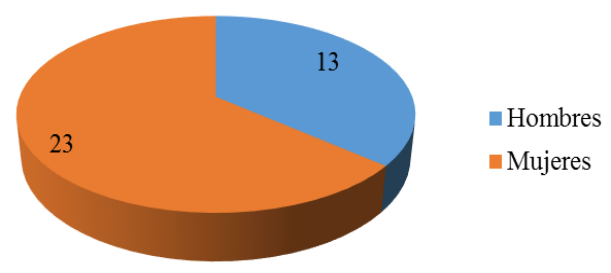

Como el gráfico nos refleja la mayor parte de las personas mayores sometidas a algún tipo de maltrato fueron mujeres. El $36 \%$ de los sujetos sometidos a alguna situación de maltrato fueron hombres (13 sujetos) frente al 64\% de mujeres (23 sujetos). Este resultado nos puede llevar a errores de interpretación si no tenemos en cuenta que la mayoría de personas que viven en residencias, siguiendo la estela de los datos de tipo sociodemográfico respecto a esperanza de vida, son mujeres.

\section{Estado civil}

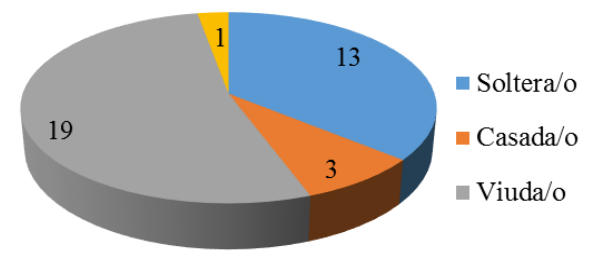

Los resultados del estudio nos señala que el estado civil de la persona mayor sometida a alguna situación de maltrato no institucional es viudedad con un $53 \%$ del total (19 sujetos), seguido de la soltería con un 36\% de sujetos entrevistados (13 sujetos). Al igual que ocurría con el sexo no puede interpretarse este resultado a la ligera ya que 
las estadísticas sociodemográficas publicadas al efecto arrojan que el estado civil de la población mayor en nuestro país es precisamente la viudedad y la soltería (Del Barrio y Abellán, 2009). Por ello estos datos solo reflejan una confirmación del muestra respecto al total de la población estudiada.

\section{Tipo de maltrato}

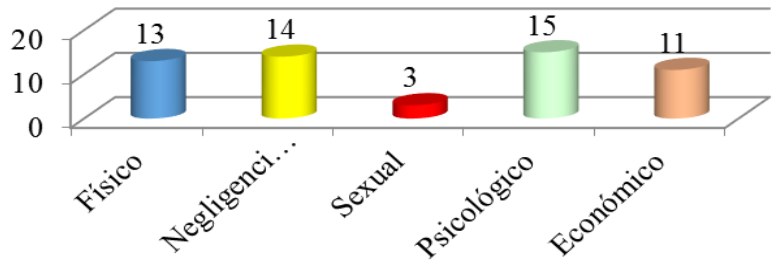

Como puede apreciarse el tipo de maltrato más prevalente está más o menos repartido entre el de tipo psicológico con un $42 \%$ del total (15 sujetos) y el de la negligencia y abandono con un 39\% (14 sujetos). A la hora de interpretar los resultados hay que tener en cuenta que los datos obtenidos son absolutos y no excluyentes, es decir, no tienen en cuenta la posibilidad de más de un tipo de maltrato en la misma persona, así pueden haber personas que padezcan maltrato psicológico y también negligencia o de otro tipo, aunque los datos no lo reflejen.

\section{Cuando se produce el maltrato (periodicidad)}

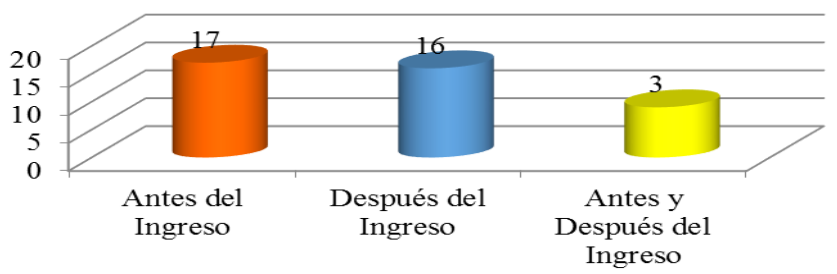

Los datos obtenidos en este apartado, son excluyentes entre sí, de manera que solo se ha tenido en cuenta el hecho de que o bien se daba antes o bien después o bien antes o después del ingreso. Es muy interesante la interpretación que podemos darle a la segunda columna, después del ingreso, ya que esta se produce precisamente si no promovida por la misma institucionalización si facilitada por la misma. En este caso el $44 \%$ de los casos (16 sujetos) de maltrato ocurren después del ingreso; casi la mitad, el $47 \%$ de los casos ocurrían antes de ingresar, los cuales se presupone que desaparecieron al ir a vivir a la residencia, lo cual se puede entender al asumir que la institucionalización era promovida precisamente por esta situación socialmente patológica. Solo un pequeño porcentaje de casos, con un $8 \%$ de los casos ( 3 sujetos) este maltrato se mantiene. 


\section{Quien ejerce el maltrato sobre el anciano}

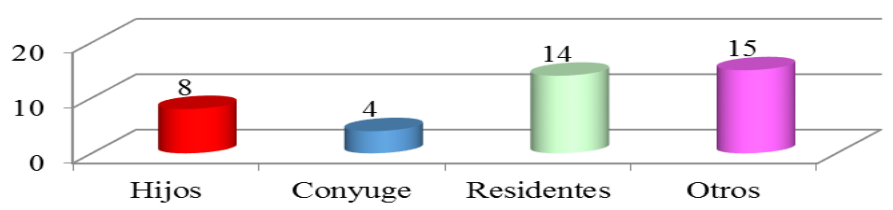

Los resultados fueron muy clarificadores ya que la mayor parte de maltratadores no solían ser los familiares más allegados, siendo en orden de prevalencia el procedente de amigos, vecinos, o familiares en grado de consanguinidad más alejados que los hijos y cónyuge (sobrinos) con un $42 \%$ de los casos (15 sujetos) seguido del procedente de otros residentes con un nada despreciable 38\% (14 sujetos). Es muy interesante el dato referido a los otros residentes ya que si bien estos no son familiares, ni personal de la residencia, si viven en el mismo lugar que la persona mayor maltratada. Es muy importante tener en cuenta estos datos ya que las relaciones interpersonales son siempre fruto de controversia y más todavía cuando comparten un mismo espacio.

\section{DISCUSIÓN Y CONCLUSIONES}

Hemos de señalar que conforme se han ido exponiendo los resultados hemos ido comentando y discutiendo las posibles interpretaciones que estos podrían tener sobre todo en el ámbito institucional. Por ello, procederemos a exponer las conclusiones.

Primero. El gerotipo de persona mayor maltratada es mujer de unos 80 años de edad, que no tiene cónyuge (viuda o soltera), que padece vejaciones de índole psicológica, y negligentes, por parte de familiares no allegados (sobrinos la mayoría) y por otros residentes, y que suelen estar ya presentes en más de la mitad de los casos antes del ingreso aunque se interrumpen al hacerse efectivo este. Este gerotipo nos ha permitido identificar sujetos de riesgo fácilmente identificables al ingreso en la residencia lo cual permite desarrollar protocolos de actuación y abordaje multi e interdisciplinar para prevenir su aparición o disminuir su existencia.

Segundo. La aparición de resultados bastante prevalentes sobre maltrato después del ingreso nos hace pensar más en aquel procedente de otros residentes, por lo que deberíamos hacer reflexión interna acerca del papel que juegan las organizaciones que acogen a ancianos en sus centros respecto a la prevención de este tipo de maltrato así como del abordaje del mismo.

Tercero. La riqueza de los datos cuantitativos es limitada. Si bien se podría apurar un poco más los datos recogidos procesándolos con programas que nos permitieran cruzar las variables este análisis es previsiblemente finito. Sería muy interesante una aproximación cualitativa de este problema protagonizada por la 
entrevista focalizada que buscara aprehender los matices y variables socioculturales que inciden tanto en la presencia como en la persistencia de esta situación desagradable e indigna para el anciano.

\section{REFERENCIAS}

AAVV (2007). Malos tratos a personas mayores. Madrid: Imserso. Disponible en: http://www.imserso.es/InterPresent1/groups/imserso/documents/binario/jmalostratosint.p df.

Comunidad Autónoma de la Región de Murcia (2005, 3 de Junio). Decreto 69/2005: por el que se establecen las condiciones mínimas que han de reunir los centros residenciales para personas mayores de titularidad pública o privada. Boletín Oficial de la Región de Murcia de 13 de Junio de 2005 no $133,14167-14181$.

Del Barrio, E. y Abellán, A. (2009). "Indicadores demográficos” en CSIC: Informe: 2008. pp 3366. Barcelona: Fundación IGEMA.

Delgado, A. (2012). Cuidador geriátrico no profesional. En M. García y R. Martínez, Enfermería y envejecimiento. Barcelona: Elsevier Masson.

Espiniella, B., Goyenechea, J., Pinilla, A. y Sánchez, C. (2013). Trabajo en grupo para la promoción del buen trato a mujeres mayores. Madrid: Unión de Asociaciones Familiares.

Goffman, E. (1970). Internados. Buenos Aires: Amorrortu.

Rodríguez, P. (2007). Residencias y otros alojamientos para personas mayores. Madrid: Panamericana.

Recibido: 30 de abril de 2014 Recepción Modificaciones: 26 de mayo de 2014

Aceptado: 2 de junio de 2014 